\title{
Towards effective capacity planning in a perinatal network centre
}

\author{
Md Asaduzzaman ${ }^{1}$, Thierry J Chaussalet ${ }^{1 *}$, Shola Adeyemi ${ }^{1}$, Salma Chahed ${ }^{1}$, \\ Jane Hawdon ${ }^{2,3}$, Daniel Wood ${ }^{3}$, Nicola J Robertson ${ }^{2,3}$ \\ ${ }^{1}$ Health and Social Care Modelling Group, Department of Information Systems and Computing, School of \\ Electronics and Computer Science, University of Westminster, London, UK \\ ${ }^{2}$ EGA UCL Institute for Women's Health, University College London, London, UK \\ ${ }^{3}$ North Central London Perinatal Network, London, UK \\ ${ }^{*}$ corresponding author
}

Keywords: health service, queueing model, overflow, admission rejection, neonatal unit

\begin{abstract}
Objective: To study the arrival pattern and length of stay in a neonatal intensive care/high dependency unit (NICU/HDU) and special care baby unit (SCBU) and the impact of capacity shortage in a perinatal network centre. To provide an analytical model for improving capacity planning.
\end{abstract}

Methods: The data used in this study have been collected through the South England Neonatal Database (SEND) and the North Central London Perinatal Network Transfer Audit between 1 January and 31 December 2006 for neonates admitted and refused from the neonatal unit at University College London Hospital (UCLH). Exploratory data analysis was performed. A queueing model is proposed for capacity planning of a perinatal network centre.

Outcome measures: Predicted number of cots required with existing arrival and discharge patterns; impact of reducing length of stay.

Results: In 2006, 1002 neonates were admitted to the neonatal unit at UCLH, 144 neonates were refused admission to the NICU and 35 to the SCBU. The model shows the NICU requires 7 more cots to accept $90 \%$ neonates at the NICU. The model also shows admission acceptance can be increased by $8 \%$ if length of stay can be reduced by 2 days.

Conclusion: The arrival, length of stay and discharge of neonates having gestational age $<27$ weeks were the key determinants of capacity. The queuing model can be used to determine the cot capacity required for a given tolerance level of admission rejection.

\section{INTRODUCTION}

Every year over 80,000 (approximately 10\%) neonates are born premature, very sick, or very small and require some form of specialist support in the United Kingdom ${ }^{1,2}$. Neonatal services aim to offer high quality care for these vulnerable babies. Over a sixmonth period in 2006-07, neonatal units were shut to new admissions for an average of 24 days $^{2}$. One in ten units exceeded its capacity for intensive care for more than 50 days during the six-month period ${ }^{2}$. Therefore, capacity planning is a major concern for all neonatal units in the UK. 
Neonatal care is provided in specialist units that are graded into three levels set by the British Association of Perinatal Medicine ${ }^{3}$ (BAPM). A level 1 unit provides special care which is the least intensive and most common type of care. In this unit, neonates may need to be fed through a tube, be supplied with extra oxygen or treated with ultraviolet light for jaundice. In a level 2 unit, neonates may also receive high dependency care such as breathing via continuous positive airway pressure or intravenous feeding, and short-term intensive care. A level 3 unit provides the whole range of medical neonatal care: special care, high dependency care, and intensive care. In intensive care, neonates will often be on a ventilator and need constant care to be kept alive. It is a national recommendation that neonates with complex needs or requiring long periods of respiratory support have their initial care in a level 3 unit.

\section{What is already known on this topic?}

Neonatal units in the UK are rejecting a huge number of neonates due to capacity shortage.

\section{What this study adds?}

A queueing model is developed for capacity planning of a neonatal unit in the UK. A substantial number of NICU cots are required to achieve $95 \%$ neonates to be admitted at UCLH neonatal unit.

Managed clinical networks for neonatal care were established in 2004 to achieve an appropriate level of care in the right place ${ }^{4}$, and have for basic aim to provide all levels of care for $95 \%$ of mothers and neonates within the network area where they live. A neonatal/perinatal network is organised in such a way that there is at least one level 3 unit which closely works with other level 2 and level 1 units. The 182 neonatal units established in England are organized through 24 networks ${ }^{5}$. In London 5 neonatal networks were set up to coordinate and manage neonatal care. The North Central London Perinatal Network (NCLPN) consists of six neonatal units based at Barnet, Chase Farm, Whittington, Royal Free, Great Ormond Street and University College London Hospital (UCLH), which is the only level 3 unit in the network and referred to as the perinatal network centre.

Queueing models are among the most popular and suitable health care modelling techniques for stochastic systems. Many queueing models, found in the literature, have been used to determine bed requirement and allocation, staff and manpower planning, resource utilisation and planning, patient flow, etc ${ }^{6}$. With a minimum data requirement (random arrival pattern and length of stay), these models can give immediate estimates of quantities such as the number of beds required for given levels of service, and immediate answers to many 'what if' scenarios. Another important aspect of queueing models is that they can be easily implemented as a user-friendly decision support tool, for instance, within a spreadsheet environment.

This study tries to discover the potential impact of capacity shortage of a perinatal network centre and aims to develop a queueing model-based capacity planning tool for a neonatal unit considering the proportion of rejected admissions. 


\section{METHODS}

\section{Settings and patients}

This study concerns the UCLH level 3 neonatal unit, which comprises a neonatal intensive care unit (NICU) and a special care baby unit (SCBU). The NICU provides neonatal intensive care and high dependency care, while the SCBU provides special care. In 2006, UCLH consisted of 12 NICU cots and 15 SCBU cots. Temporary care was also provided in 8 transitional care (TC) cots.

The data have been collected through the South England Neonatal Database (SEND) and the North Central London Perinatal Network Transfer Audit between 1 January and 31 December 2006. The SEND database contains information about neonates cared for at any neonatal unit in the South England area including UCLH. The data provide comprehensive and accurate information about each neonate's stay in the NICU and SCBU, including dates and times of arrival/discharge, source and reason of admission, birth weight and gestational weeks. But it does not include information on neonates whose admission requests were rejected. The NCLPN Transfer Audit contains records of requests for admission and of transfers of mothers and neonates throughout the network. Therefore, to explore the actual arrival pattern, information on all neonates admitted to the neonatal unit at UCLH between 1 January and 31 December 2006, has been merged with the NCLPN Transfer Audit data. Agreement from the Caldicott Guardian was obtained from each Trust. The study involved data collected solely from secondary sources and fully anonymised, hence did not require ethics committee approval.

All 1002 neonates (SEND database) cared for at the UCLH neonatal unit in the year 2006 were considered eligible for this study. Of these 1002 neonates, $31 \%$ were cared for at the intensive care and high dependency units and the remaining were cared for at the special care unit. During the year, at least 144 neonates $(33 \%)$ were refused admission to NICU and $35(4 \%)$ to SCBU.

\section{Mathematical analyses}

Exploratory data analysis was performed to discover the sources of admission of neonates, their booked place of delivery, place of birth, reasons for admission and discharge destination. Patterns of admission requests and length of stay of neonates for each category of care have been estimated. A queueing model was developed to study the influence of cot capacity, admissions and length of stay on admission rejection.

\section{RESULTS}

\section{Activity of UCLH in $\mathbf{2 0 0 6}$ and impact of capacity shortage}

Of the 1002 neonates admitted at UCLH in 2006, 83\% had UCLH as their booked place of delivery, $7 \%$ were booked in other neonatal units within the network, and $10 \%$ came from outside the network (Table 1). So, $90 \%$ babies admitted at UCLH are covered by the network. The place of birth of $95 \%$ of these neonates was UCLH since mothers expected to give birth to neonates with $<27$ gestational age are transferred to UCLH. Of the 1002 neonates, 39\% were admitted for neonatal intensive or high dependency care (NICU), $40 \%$ for special care (SCBU), and $21 \%$ for transitional care (TC). Table 2 shows the length of stay (LoS) of neonates at NICU and SCBU. Although the mean LoS was 14 days at the NICU and 8 days at the SCBU, few neonates stayed up to 127 days at the $\mathrm{NICU}$ and 52 days at the SCBU. 
Table 1 Main characteristics of the study population $(n=1002)$

\begin{tabular}{|l|l|}
\hline Booked place of delivery & 90 \\
Hospitals within NCLPN & 83 \\
UCLH & 7 \\
Other hospitals & 10 \\
Hospitals outside NCLPN & \\
\hline Place of birth & 95 \\
UCLH & 5 \\
Others & \\
\hline Admission & 39 \\
NICU & 40 \\
SCBU & 21 \\
\hline TC & \\
\hline Discharge destination from NICU & 66 \\
SCBU & 34 \\
\hline Discharged &
\end{tabular}

Table 2 Length of stay pattern of neonates at NICU and SCBU

\begin{tabular}{|l|l|}
\hline Length of stay & Days \\
\hline NICU & \\
Max. & 127 \\
Mean & 14.1 \\
SD & 20.3 \\
SCBU & \\
Max. & 52 \\
Mean & 7.8 \\
SD & 7.6 \\
\hline
\end{tabular}

Figure 1 and 2 show monthly admission and rejection patterns at the NICU and the SCBU in 2006. A high number of admission requests (33\%) were rejected from the NICU throughout the year; this suggests a significant and steady capacity shortage. A lower rejection level (5\%) is observed at the SCBU. Among rejected babies at NICU, more than $60 \%$ were from outside the network, and at SCBU-TC, $82 \%$ were from network area and the rest (18\%) from outside network. Due to the random nature of arrival and length of stay of neonates at the unit, cot occupancy is also random. Figure 3 and 4 show the weekly mean number of cots occupied at the NICU and the SCBU. Figure 3 shows that the UCLH NICU had to run beyond its 12-cot capacity more than half the year. Figure 4 shows that all 15 funded special care cots were occupied most of the year and the 8 transitional care cots were used frequently. It is clear from Figures 1-4 that neonates were overflowed and a considerable number of neonates were rejected from UCLH neonatal unit due to overall capacity shortage. 


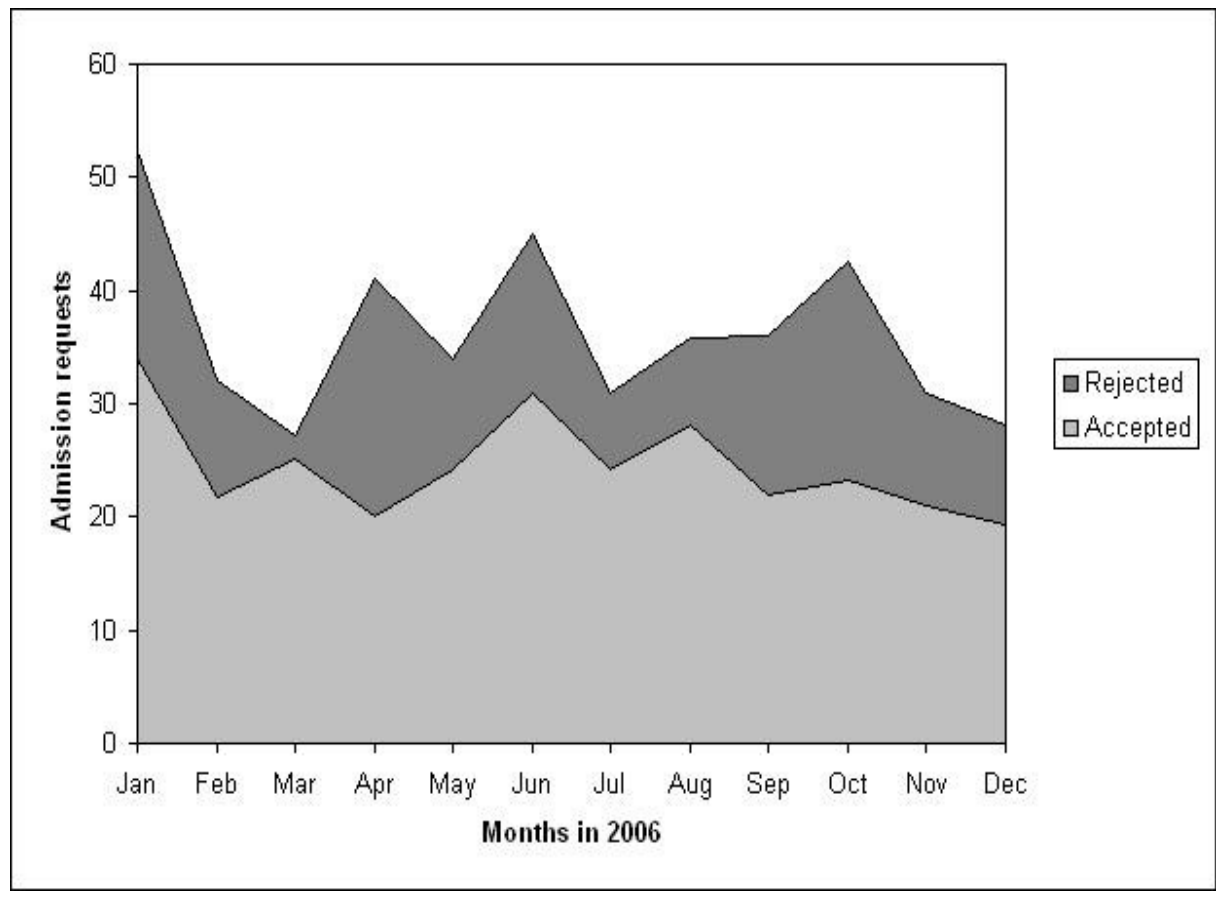

Figure 1 Area curve for rejected admission requests and neonates cared at UCLH NICU.

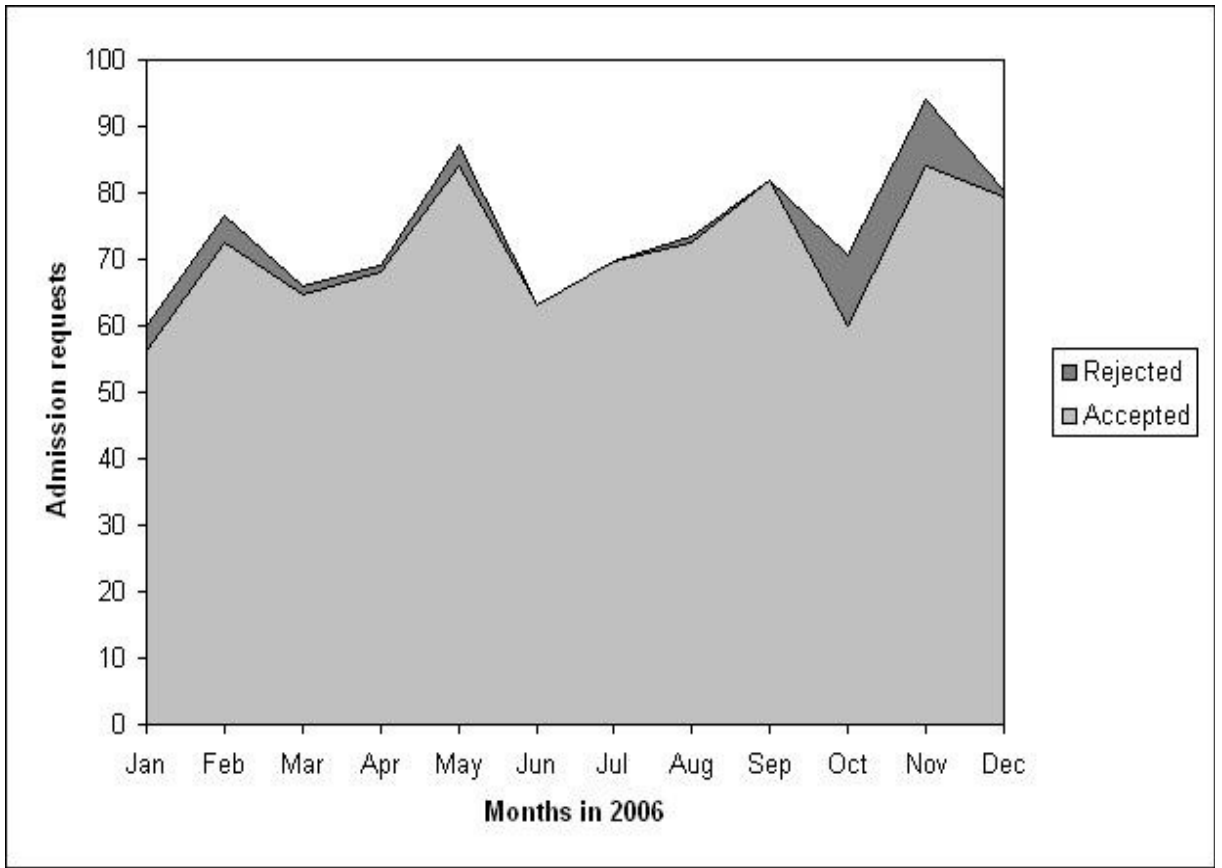

Figure 2 Area curve for rejected admission requests and neonates cared at UCLH SCBU. 


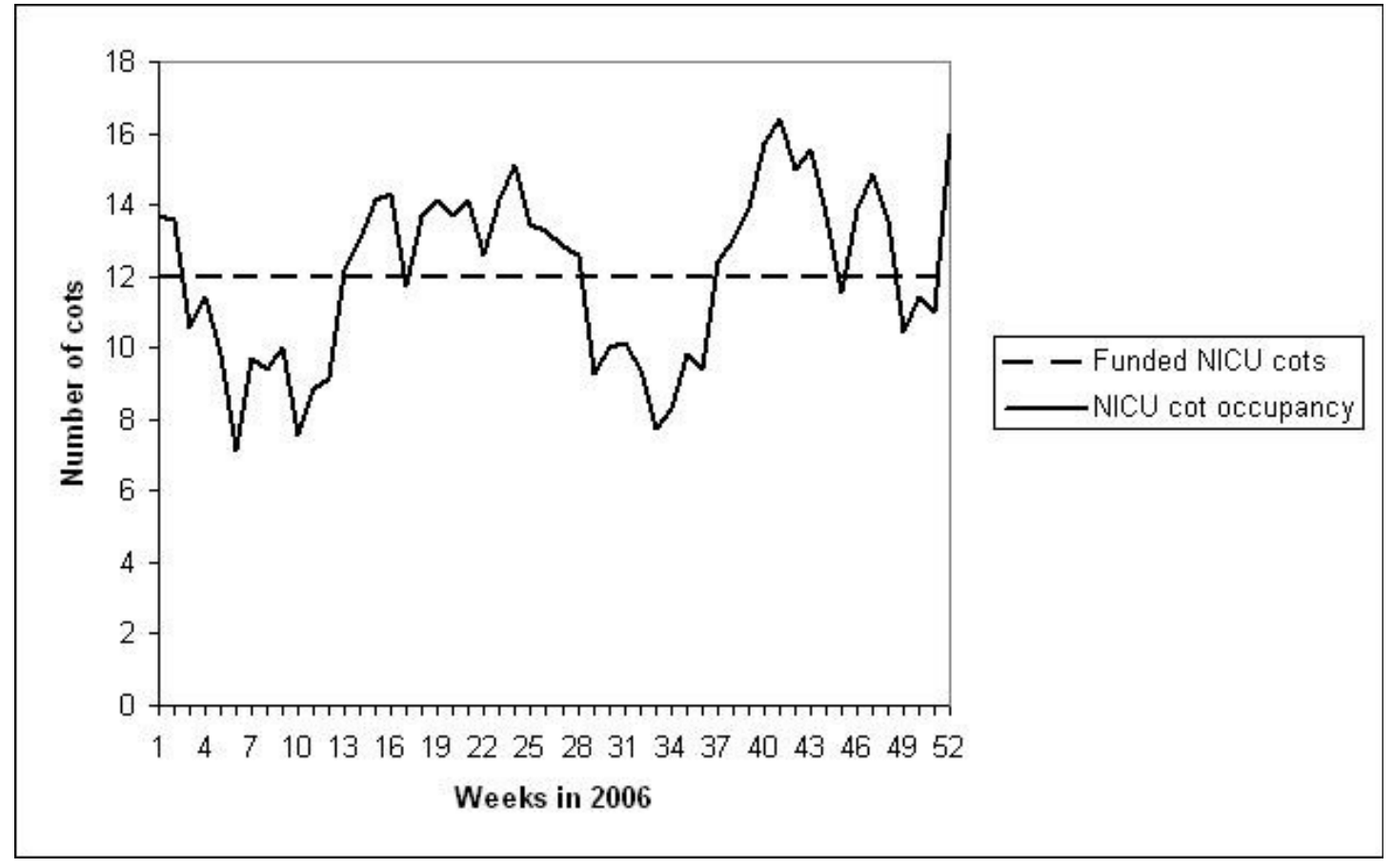

Figure 3 Weekly mean number of cots occupied and number of funded cots at UCLH NICU.

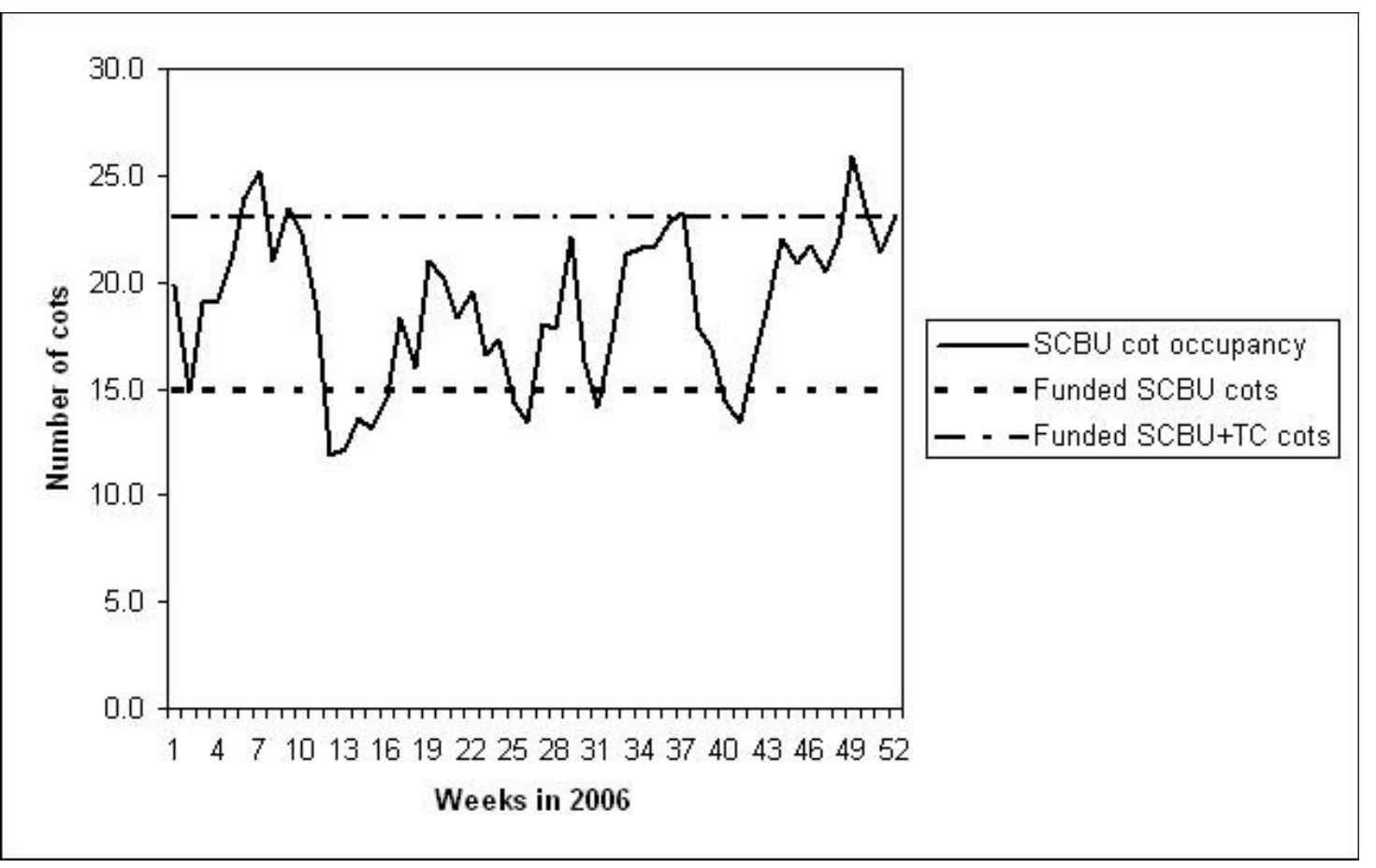

Figure 4 Weekly mean number of cots occupied and number of funded cots at UCLH SCBU and TC. 


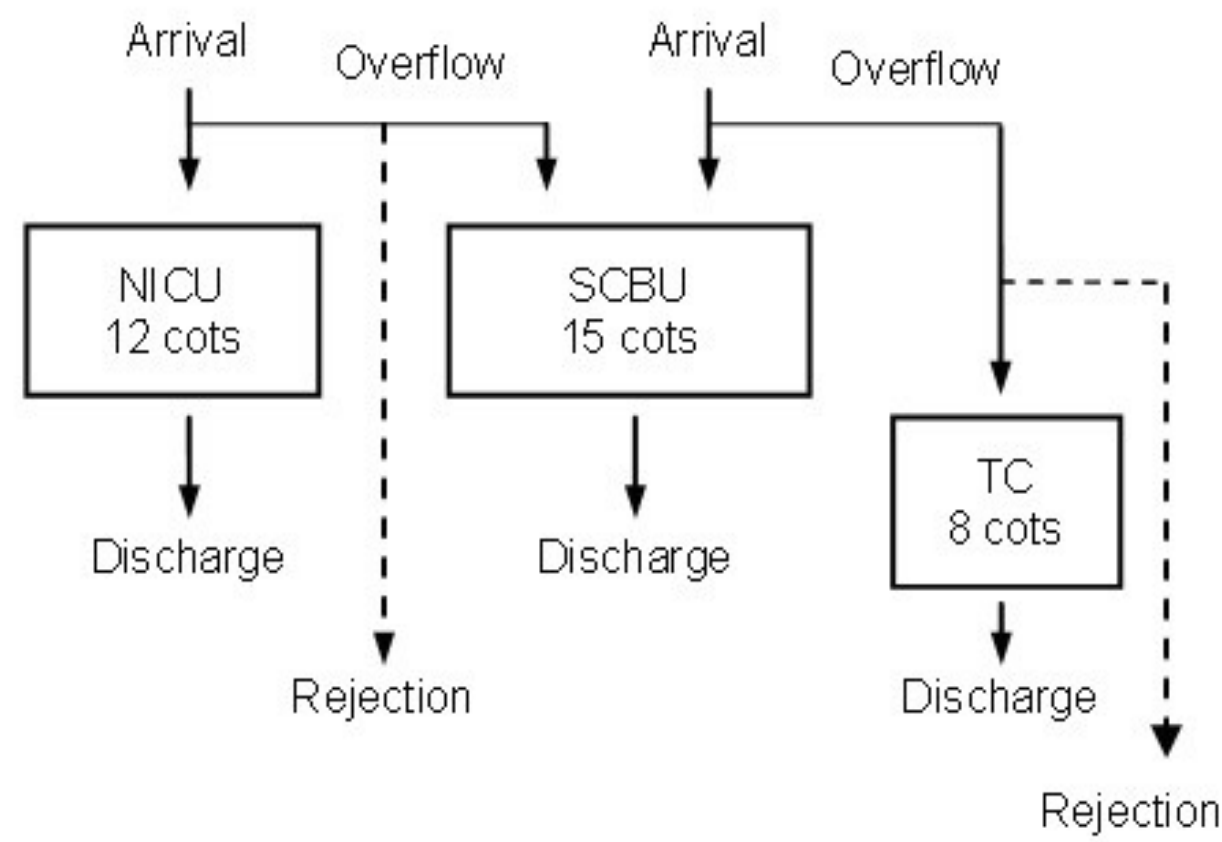

Figure 5 Proposed model for the UCLH NICU-SCBU system.

\section{Modelling UCLH and analysis for capacity planning}

A queueing network model is used for capacity planning of the UCLH neonatal unit based on a framework developed by Asaduzzaman et al ${ }^{6}$. Figure 5 shows the proposed model for UCLH with NICU and SCBU. A detailed flow of neonates is also demonstrated in the figure. An admission request can be either accepted or rejected based on the availability of cots. To capture overflow, we assume that when all cots are occupied at $\mathrm{NICU}$, then a neonate requiring intensive or high dependency care overflows to SCBU only if a SCBU cot is available and eventually brought back to NICU upon availability of a NICU cot. Similarly a neonate requiring special care will be overflowed to TC depending upon demand and circumstances if all SCBU cots are blocked.

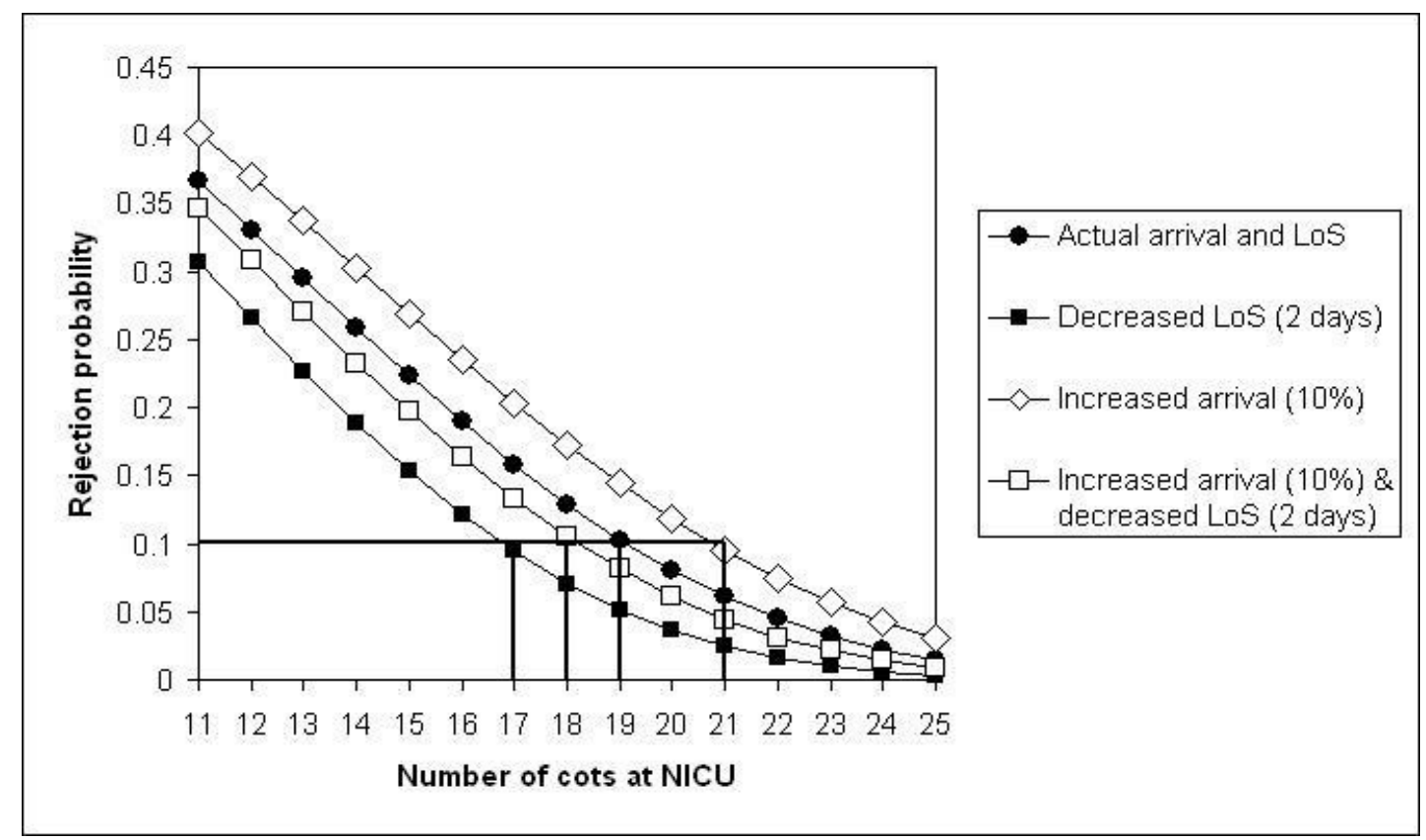

Figure 6 Rejection probability against number of cots at NICU. 


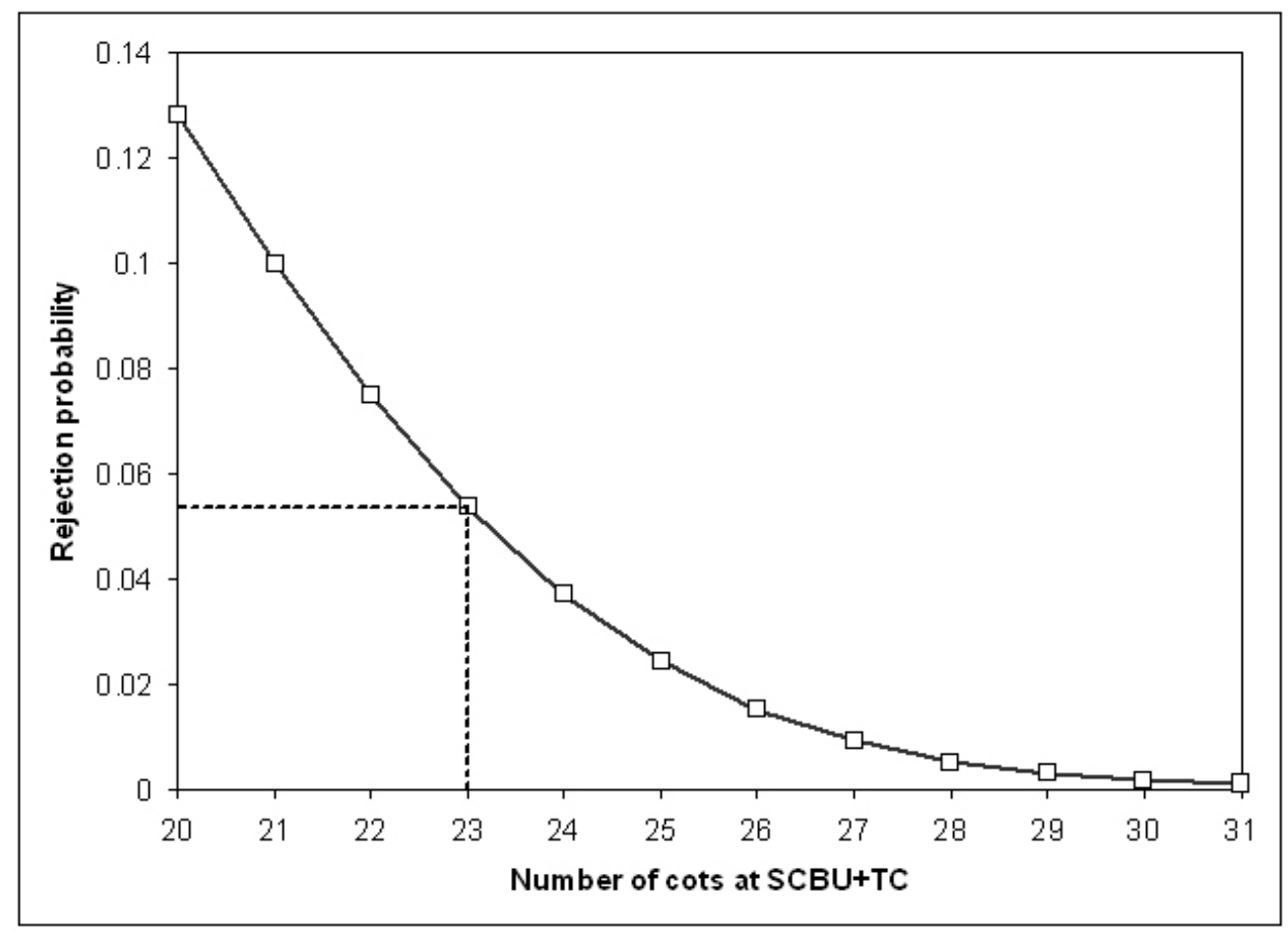

Figure 7 Rejection probability against number of cots at SCBU+TC.

Based on these assumptions using the model, we derive the admission rejection probability for different number of cot combinations for all categories of care. The decision on number of cots can be made considering their corresponding admission rejection probability.

Figure 6 shows the admission rejection probability at NICU against the number of cots, and also presents different scenarios. The figure indicates that, with 12 cots, the probability that the NICU will reject an admission request is 0.33 . In order to accept $90 \%$ of the admission requests, 7 extra cots are required. The figure also explores the effect of shortening the average LoS at NICU by 2 days - assuming this is not detrimental to the quality of care. The model shows that, with an average LoS reduction of 2 days, $7 \%$ more neonates could be accepted at NICU with the current 12 NICU cots, while, to accept $90 \%$ of admission requests, 5 extra cots would be sufficient instead of 7 . With a $10 \%$ increase in the arrival rate, 9 extra cots would be required with the current average LoS, while 6 extra cots would be sufficient if the average LoS could be reduced by 2 days. Figure 7 shows the admission rejection probability at SCBU against the number of cots.

Table 3 Comparison of rejection probabilities between models

\begin{tabular}{|l|l|l|l|l|}
\hline & $\begin{array}{l}\text { Cot } \\
\text { capacity }\end{array}$ & $\begin{array}{l}\text { Observed } \\
\text { Rejection } \\
\text { probability }\end{array}$ & \multicolumn{2}{|c|}{ Estimated rejection probability } \\
\cline { 3 - 5 } & Standard model & & Proposed model \\
\hline NICU & 12 & 0.325 & 0.377 & 0.333 \\
SCBU (TC) & $15(8)$ & 0.038 & 0.070 & 0.056 \\
\hline
\end{tabular}

${ }^{*}$ As in Asaduzzaman and Chaussalet (2008) ${ }^{7}$. 
Table 3 summaries the rejection probabilities for the NICU and SCBU+TC. With the existing $15 \mathrm{SCBU}$ and $8 \mathrm{TC}$ cots, the rejection probability is 0.056 at SCBU. The rejection probability estimated with the model matches the observed rejection probability.

\section{DISCUSSION}

Our findings from the data analyses clearly show that UCLH neonatal unit has rejected a huge number of admission requests. The majority of these are from NICU and a small number from SCBU. The proposed model takes the issue of overflow into account while deriving the required number of cots for any specified level of admission request rate in both levels. The model shows that 7 more cots are required at NICU to be able to accept $90 \%$ of admission requests at UCLH NICU. Table 3 shows the comparison of model results with the observed values. The estimated rejection probabilities are close to the observed rejection probabilities which imply the proposed model works accurately and can be used to determine the number of cots required for each level of care in a neonatal unit. Another important aspect of the model is its application for testing different scenarios. For instance, with the existing 12 NICU cots, $7 \%$ more neonates could be admitted to NICU if the same high level of care could be provided in 2 days less than the current average time. Similarly, other scenarios can also be tested. We could change the arrival patterns at all levels of care and determine the required number of cots.

Interestingly, the UCLH neonatal unit has moved at the end of 2008 to larger facilities and now has 15 SCBU cots (same as in 2006) and 17 NICU cots (5 more than in 2006). The model could be used to estimate the impact of this increased capacity on the rejection probability under various scenarios. For instance, assuming no change in arrival rate and average LoS, with $17 \mathrm{NICU}$ cots the rejection probability at NICU is expected to drop from 0.3250 to around 0.17 while with 22 NICU cots, it would drop to under 0.05 .

We believe the proposed model would help with solving capacity problem currently faced by many neonatal units in the UK. Although the model formulation has been developed for a specific perinatal network centre, it can be used for any level 3 (perinatal network centre) or level 2 units since their structures are similar. It can easily be extended to handle a completely separate HDU. In brief, the proposed model can serve as an important capacity planning tool for any neonatal unit. To achieve $95 \%$ target for the care of mothers and babies within the network area, some perinatal network centres or level 2 units often admit babies booked at units within the network, even when they are full. The model used here depends on a fairly uniform approach to arrivals of neonates and their movement between the various levels of care. Future work will consider the use of priority queueing models to take into account differences in arrivals and movements of babies according to their booked place of delivery. Seasonal or periodic variations in demand could not be confirmed with only one year of data. However, with more data available, they are likely to become visible. The models could be modified by adjusting arrival and LoS patterns for seasonal and periodic variations.

This paper emphasises capacity planning for neonatal units in the UK to reduce the level of rejected admissions, which often result in high risk of neonatal mortality and extra cost. The findings of this paper should be of interest to the Department of Health $(\mathrm{DH})$, health care researchers, neonatologists and perinatal network managers. 


\section{ACKNOWLEDGEMENTS}

We thank the network manager and nurses in the NCLPN, especially Olga Kurtinyank in Royal Free neonatal unit, and also all mothers and neonates cared at UCLH.

\section{Competing interests}

The authors wish to declare that extramural funding, directed the delivery of this project or the writing of this manuscript, was not provided by any company or granting agency. Support was provided solely from institutional and/or departmental sources.

\section{REFERENCES}

1. Department of Health. Report of the neonatal intensive care services review group, 2003.

2. Bliss. Special delivery or second class: are we failing special care babies in the UK? Bliss - The Premature Baby Charity, 2007.

3. British Association of Perinatal Medicine (BAPM). Standards for hospitals providing neonatal intensive and high dependency care (Second Edition) and Categories of Babies Requiring Neonatal Care, 2001.

4. Marlow N, Gill, AB. Establishing neonatal networks: the reality, Arch. Dis. Child. Fetal Neonatal Ed. 2007, 92:F137-42.

5. Redshaw M, Hamilton K. A survey of current neonatal unit organisation and policy, Bliss - The Premature Baby Charity and National Perinatal Epidemiology Unit, University of Oxford, 2005.

6. Asaduzzaman M, Chaussalet TJ, Robertson NJ. A loss network model with overflow for capacity planning in neonatal unit, Ann. Oper. Res., 2009, Published online, DOI 10.1007/s10479-009-0548-x.

7. Asaduzzaman M, Chaussalet TJ. Modelling and performance measure of a perinatal network centre in the United Kingdom, In Proc. 21th IEEE Intl. Sym. Com. Based Med. Sys., 2008, 506-11. 